\title{
CRISPR/Cas9 directed to the Ube3a antisense transcript improves Angelman syndrome phenotype in mice
}

\author{
Ralf S. Schmid, Xuefeng Deng, Priyalakshmi Panikker, Msema Msackyi, Camilo Breton, and James M. Wilson \\ Gene Therapy Program, University of Pennsylvania Perelman School of Medicine, Philadelphia, Pennsylvania, USA.
}

\begin{abstract}
Gene editing holds the potential to correct mutations and cure devastating genetic disorders. The technology has not yet proven efficacious for therapeutic use in CNS diseases with ubiquitous neuronal defects. Angelman syndrome (AS), a severe neurodevelopmental disorder, is caused by a lack of maternal expression of the UBE3A gene. Because of genomic imprinting, only neurons are affected. One therapeutic approach focuses on the intact paternal UBE3A copy in patients with AS that is silenced by an antisense transcript (UBE3A-ATS). We show here that gene editing of Ube3a-ATS in the mouse brain resulted in the formation of base pair insertions/deletions (indels) in neurons and the subsequent unsilencing of the paternal Ube3a allele in neurons, which partially corrected the behavioral phenotype of a murine AS model. This study provides compelling evidence to further investigate editing of the homologous region of the human UBE3A-ATS because this may provide a lasting therapeutic effect for patients with AS.
\end{abstract}

\section{Introduction}

Angelman syndrome (AS) is a neurodevelopmental disorder characterized by intellectual disability, developmental delay, speech impairment, ataxia, and seizures $(1,2)$. There is no cure available. This disorder is caused by maternal deficiency of the neuronal imprinted gene ubiquitin-protein ligase E3A (UBE3A), which results in a lack of UBE3A protein expression selectively in neurons (3-5). About $70 \%$ of patients with AS carry a large deletion on chromosome 15 that includes the $U B E 3 A$ locus. However, codeletion of genomic regions beyond $U B E 3 A$ may contribute to the AS phenotype as well (6). Patients with AS typically carry an intact paternal $U B E 3 A$ allele that is silenced by a nuclear long noncoding mRNA, the UBE3A antisense transcript (UBE3A-ATS), which extends across most of the UBE3A gene locus (7-9). Studies show that interfering with murine Ube3a-ATS results in paternal Ube3a expression $(8,10,11)$. Applying these approaches in maternal Ube3a-deficient mice leads to paternal Ube3a expression in the CNS, thus restoring Ube 3 a expression and amelioration of the mouse AS phenotype. Genetic restoration of Ube3a expression in Ube3a-KO mice demonstrated the greatest efficacy to correct the neurobehavioral phenotype when accomplished during early postnatal development (12). Although the mechanisms that lead to paternal Ube3a expression after perturbing Ube3a-ATS remain unclear, the effect has shown enough promise to support a clinical trial harnessing the potential power of

Conflict of interest: JMW is a paid advisor to and holds equity in Scout Bio and Passage Bio; he holds equity in Surmount Bio; he also has sponsored research agreements with Albamunity, Amicus Therapeutics, Biogen, Elaaj Bio, FA212, Janssen, Moderna, Passage Bio, Regeneron, Scout Bio, Surmount Bio, and Ultragenyx, which are licensees of University of Pennsylvania technology. JMW, CB, and RSS are inventors on patents/patent applications filed by the University of Pennsylvania. Copyright: () 2021, American Society for Clinical Investigation.

Submitted: July 22, 2020; Accepted: December 29, 2020; Published: February 15, 2021. Reference information: J Clin Invest. 2021;131(5):e142574.

https://doi.org/10.1172/JCl142574. an intact paternal allele (ClinicalTrials.gov NCT04259281). Preliminary data from this trial demonstrated significant improvement of clinical outcomes in 5 patients with AS. The treatment under clinical investigation relies on antisense oligonucleotides (ASOs) that require frequent intrathecal readministration; ASO treatment-related toxicity was observed. We aimed to evaluate the feasibility of an alternative approach to achieve longlasting therapeutic efficacy with a single intervention through gene editing of the Ube3a-ATS. We show here that introducing indels between the Ube3a 3' UTR and the Snord115 cluster (Figure 1A) interfered with the extension of the Ube3a-ATS across the Ube $3 a$ gene locus, triggering lasting expression of the Ube $3 a$ protein from the paternal allele and improving neurobehavioral symptoms in a Ube3a maternal KO mouse model for AS.

\section{Results and Discussion}

To introduce indels into Ube3a-ATS, we initially screened 12 sgRNAs that target different sites within a $12 \mathrm{~kb}$ segment of the Ube3a-ATS coding sequence (Figure 1A and Supplemental Table 1; supplemental material available online with this article; https:// doi.org/10.1172/JCI142574DS1). We aimed to select targets outside of known expressed gene loci. Cas9-induced indels at the respective target sites were assessed by amplicon sequencing (Figure 1B). We then constructed an adeno-associated virus (AAV) plasmid harboring sgRNA 7 (Figure 1B and Supplemental Figure 1 ) and the $S$. aureus Cas 9 coding sequence. We used the human synapsin promoter $(13,14)$ to selectively drive Cas 9 expression in neurons in the mouse brain. Delivery of the AAV gene editing vector - which we refer to as ATS-GE - to the neonatal mouse brain via i.c.v. injection resulted in the formation of genomic indels in $14.7 \%(8.6 \%-21.7 \%)$ of all brain cells (Figure $1 C$ ). When we expressed either a nontargeting sgRNA or a nucleasedeficient Cas9 (dCas9), indel formation remained at background frequencies $(0.17 \%$ or $0.04 \%$, respectively, Figure $1 \mathrm{C})$. Indel formation was highest after neonatal i.c.v. vector delivery. The 
A

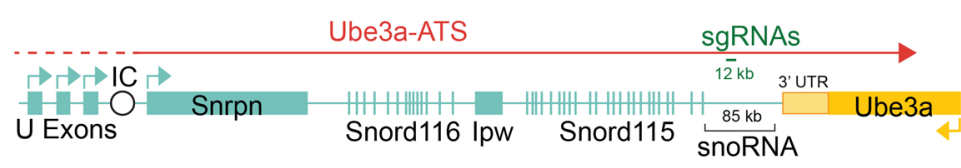

C

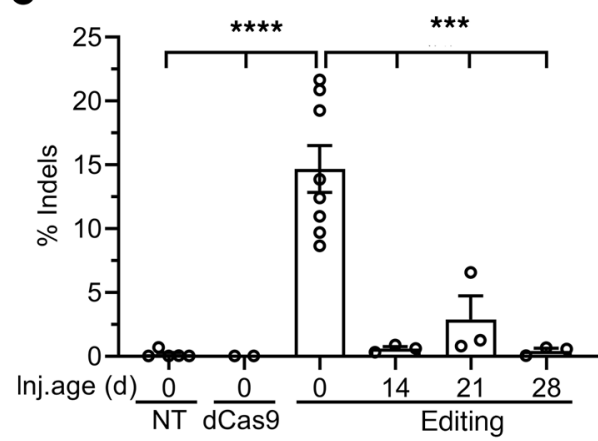

$\mathbf{G}$

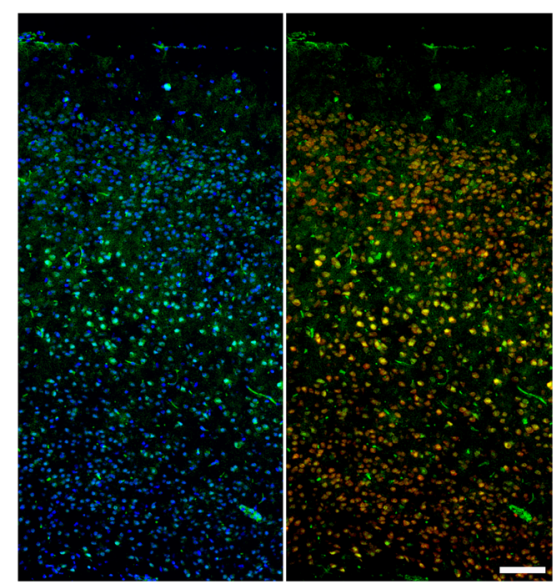

B

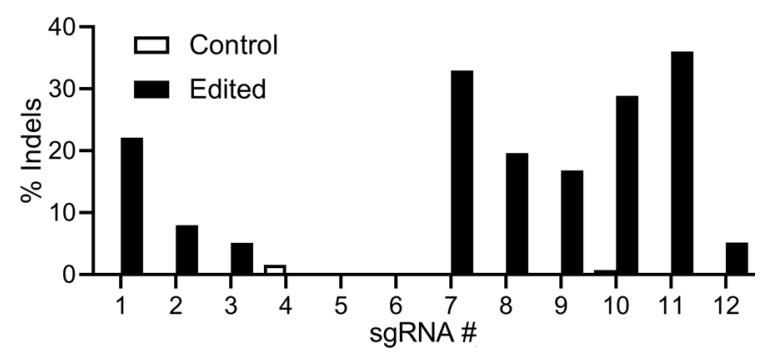

E

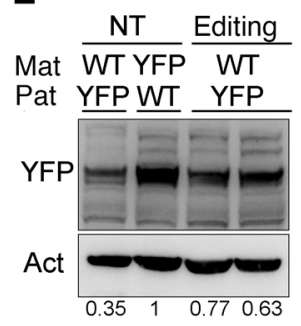

$\mathbf{F}$

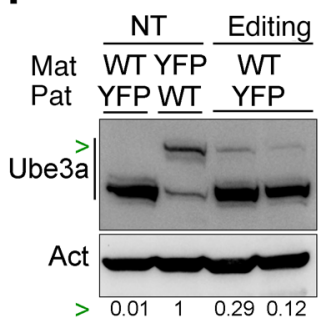

H

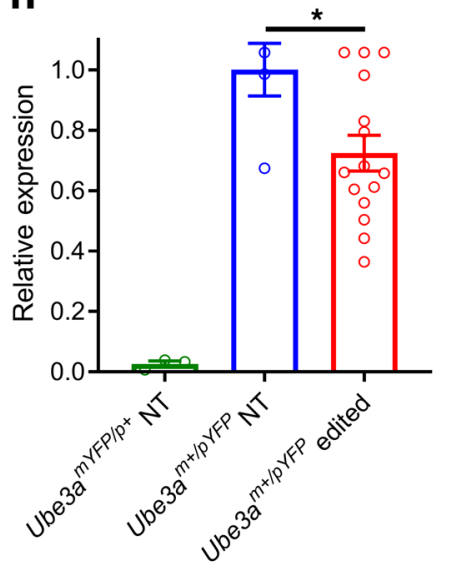

I

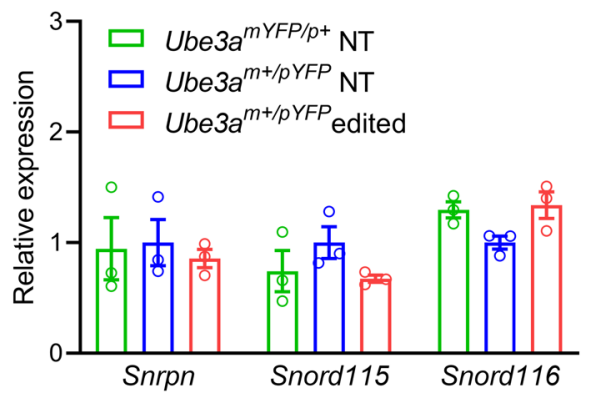

Figure 1. In vivo gene editing of Ube3a-ATS causes indel formation and expression of Ube3a-YFP reporter. (A) Schematic mouse Ube3a genomic locus (adapted from ref. 11). The region targeted in this study by sgRNAs is indicated. IC, imprinting center; snoRNA, small-nucleolar RNA. (B) In vitro indel frequencies for screened sgRNAs. (C) Ube $3 a^{m+/ p Y F P}$ mice were injected with ATS-GE vector at indicated timepoints. After 3 weeks, amplicon sequencing with cortical samples revealed an average of $14.7 \%$ of cells with indels in neonatal injected pups; the indel frequency was less than $2.9 \%$ at all other time points. Nontargeting CRISRP/Cas9 or CRISPR/dCas9 resulted in indel formation in less than $0.2 \%$ (1-way ANOVA with Tukey's pairwise comparison, $n=2$ - 6 mice/group). (D) Indels persisted in Ube3a ${ }^{m+/ p Y F P}$ neonatal mice injected with ATS-GE vector (1-way ANOVA with Tukey's pairwise comparison, $n=3-6$ mice/ group). (E and F) Representative Western blots for cortices from C demonstrate robust expression of paternal Ube3a-YFP when probed with YFP antibodies (E) or Ube3a antibodies (F). Relative quantifications normalized to actin are shown below each lane; green arrow in $\mathbf{F}$ demarcates the quantified Ube3aYFP bands. NT, nontargeting. (C) Representative immunofluorescence staining for cortices from $\mathbf{C}$ shows Ube3a-YFP expression in neurons throughout the cortex (scale bar: $100 \mu \mathrm{m})$. (H) qPCR gene expression analysis with primers specific for the Ube3a-ATS transcript. We detected a significant $28 \%$ reduction in Ube3a-ATS expression for Ube3a $a^{m+/ p Y F P}$ gene-edited cortex samples ( $n=3-15$ mice/group, 1-way ANOVA with Tukey's pairwise comparison). (I) qPCR gene expression analysis with primers specific for neighboring transcripts detected no differences in expression ( $n=3-4$ mice/group, 1-way ANOVA with Tukey's pairwise comparison, $P>0.4$ ). Means are shown with standard error; ${ }^{*} P<0.05,{ }^{* *} P<0.001,{ }^{* * *} P<0.0001$.

PHP.B capsid facilitates very efficient transduction of the mouse brain via i.v. delivery at any age (15). However, we observed much lower indel frequencies when ATS-GE was delivered at 14-28 days of age $(0.6 \%-2.9 \%$, Figure $1 \mathrm{C})$, suggesting that i.c.v. injection into the newborn mouse brain is best suited for efficient gene editing for this approach. In support of this notion, the amount of vector genome present in brain tissue after i.c.v. injection into the newborn brain was much higher (average of 2.3 genome copies [GC] per diploid genome) compared with transduction after i.v. injection at P14, P21, or P28 (0.04 to 0.19 GC per diploid genome, Supplemental Figure 2A). Additional analysis of the target site using anchored multiplexed PCR sequencing (AMP-Seq) showed that most editing events after neonatal i.c.v. delivery were indeed short indels of less than $15 \mathrm{bp}$; integration of vector sequence portions at the editing site only was observed at a frequency of $2.8 \%$ (Supplemental Figure 2B). Computational analysis predicted no additional direct matches for our chosen sgRNA throughout the mouse genome, with the nearest similarities including at least 4 


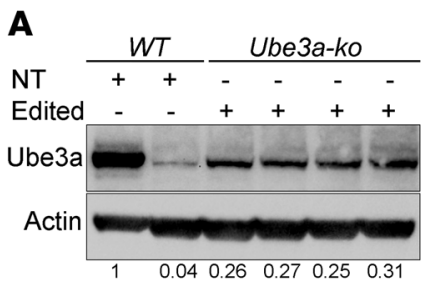

$\mathbf{F}$

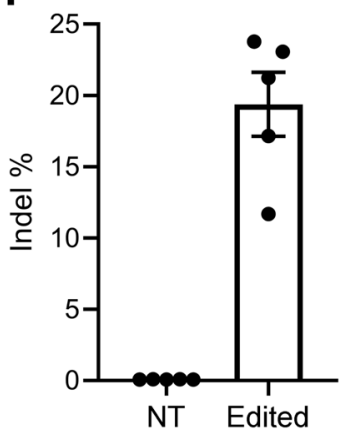

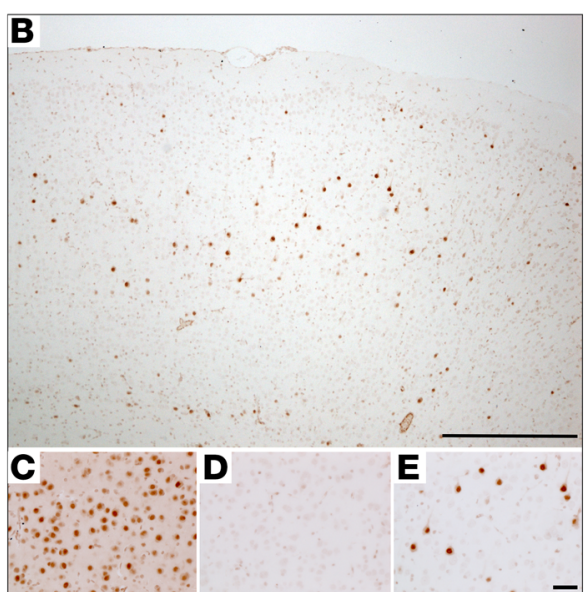

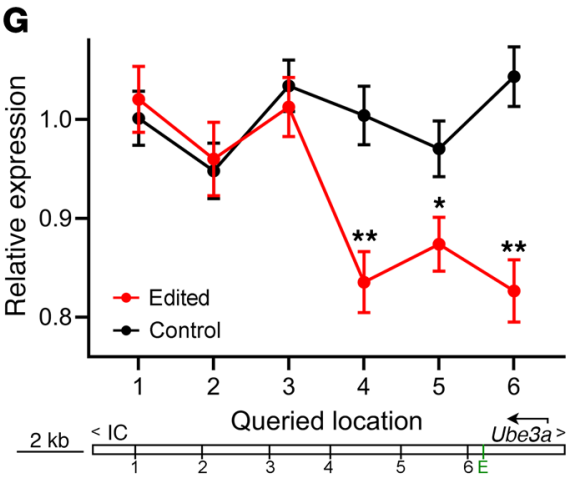

Figure 2. In vivo gene editing of Ube3a-ATS in a Ube3a$K \boldsymbol{O}$ mouse model. (A) Brains of Ube $3 a^{m-/ p+}$ mice injected with ATS-GE vector were harvested 4 months later. We detected persistent paternal Ube3a expression in the cerebral cortex by Western blotting with Ube3a antibodies. Relative quantifications of the respective Ube3a band normalized to actin are shown below each lane. (B) IHC staining of the brains from $\mathbf{A}$ with Ube3a antibodies shows paternal Ubea3a expression throughout the brain A representative cortical section is shown here (scale bar: $1 \mathrm{~mm}$ ). Magnified cortical IHC images from Ube $3 a^{m+1}$ $p_{+}(\mathbf{C})$, Ube3a $\mathrm{a}^{m-/ p_{+}}(\mathbf{D})$, and gene-edited Ube3a $\mathrm{a}^{m-/ p^{+}}(\mathbf{E})$ cortex (scale bar: $10 \mu \mathrm{m}, \mathbf{E}$ is a higher magnification of the section shown in B). (F) Amplicon sequencing analysis from the same cohort as shown in $\mathbf{A}$ revealed an average of $19.4 \%$ of cells with indels in injected pups. Injection of nontargeting CRISRP/Cas9 resulted in indel formation of $0.2 \%$ ( $n=5$ mice/group). (C) RNA extracted from cortices of the same cohort as shown in $\mathbf{A}$ was used to quantify Ube3a-ATS transcript levels at different locations between the site targeted by gene editing ( $\mathbf{E}$, about $35 \mathrm{~kb}$ distance to Ube3a $3^{\prime}$ UTR) and the imprinting center (IC). We observed a significant reduction of Ube3a-ATS starting approximately $5 \mathrm{~kb}$ away from $\mathbf{E}$ (1-way ANOVA with Tukey's pairwise comparison, $n=5$ mice/group). Means are shown with standard error; ${ }^{*} P<0.05,{ }^{*} P<0.01$. mismatches (Supplemental Table 2). Accordingly, inverted terminal repeat sequencing (ITR-Seq) revealed only a small genomewide off-target rate (1.3\%) for targeted editing with ATS-GE (Supplemental Table 3). We found that indels were present at similar frequencies when we performed molecular analyses on cortical cells at 3,8, or 14 weeks after neonatal ATS-GE vector delivery (Figure 1D). This result suggests that neurons with an edited Ube3a-ATS sequence persisted in the adult mouse brain.

Ube $3 a$-ATS interferes with the extension of the Ube3a transcript on the paternal allele, blocking Ube3a expression from the paternal allele. A promising therapeutic approach for AS relies on abrogating the extension of Ube3a-ATS across the Ube $3 a$ gene locus on the paternal allele to allow for full-length Ube $3 a$ transcript formation and thus protein expression. Thus, we next evaluated whether the observed indel formation in our studies could suppress extension of Ube3a-ATS across the Ube3a paternal allele. To unambiguously detect Ube3a expression from the paternal allele, we crossed WT females with male mice harboring an Ube3a-YFP fusion gene (16). Newborn pups i.c.v. injected with the ATS-GE vector showed expression of the Ube3a-YFP fusion protein 21 days later (Figure 1, E and F). Immunofluorescence staining revealed expression of Ube3a-YFP throughout the cortex after gene editing in $48.1 \%$ of neurons (Figure $1 \mathrm{G}$, and quantification of $13,000 \mathrm{NeuN}^{+}$cells, SEM $=8.6 \%$ ). When we replaced the targeting sgRNA in the ATS-GE vector with a nontargeting sgRNA, or replaced Cas9 with dCas9, we observed no Ube3a-YFP expression (Supplemental Figure 2, C and D). Molecular analysis revealed that $U b e 3 a-A T S$ expression from the paternal allele was reduced within the Ube3a gene locus (Figure 1H). By contrast, expression of genes located between the gene editing location and the imprinting center was unaffected (Snrpn, Snord115, or Snord116, Figure 1I). Thus, selective and efficient gene editing within the Ube3a-ATS led to Ube3a protein expression from the previously silenced paternal $U b e 3 a$ allele.

Next, we investigated whether Ube3a-ATS gene editing could drive expression of Ube3a from the paternal allele to restore Ube3a expression in neurons of maternal Ube3a-KO mice and improve the mouse AS phenotype. We administered the ATS-GE vector to neonatal maternal Ube3a-KO pups and WT littermates via i.c.v. injection. We then observed the expression of Ube3a protein throughout the brain after 4 months of incubation (Figure 2A). We detected expression of Ube3a in neurons throughout the brain (Figure 2, B-E, and Supplemental Figure 3). We observed Ube3a-positive neurons throughout the brain (Supplemental Figure $3, \mathrm{~B}$ and $\mathrm{C}$ ), and it seemed that expression was more abundant in the basal forebrain regions. A larger, more comprehensive study is needed to investigate whether expression efficiency varies among brain regions and functional substructures, but this was beyond the scope of the current investigation.

Mice that received the control AAV vector (harboring nontargeted Cas 9 or targeted $d$ Cas 9 sequences) did not show Ube $3 a$ expression from the paternal allele (Supplemental Figure 4, A and B). Molecular analysis by amplicon sequencing revealed that indel frequencies occurred at an average of $19.4 \%$ (Figure $2 \mathrm{~F}$ ), which is comparable to observations from the previous short-term study (Figure 1C). Vector integrations at the gene-editing site were low 
A

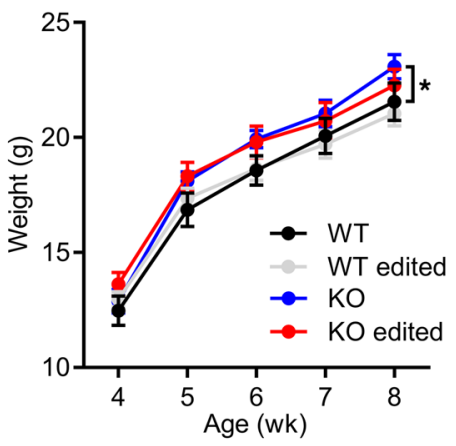

D

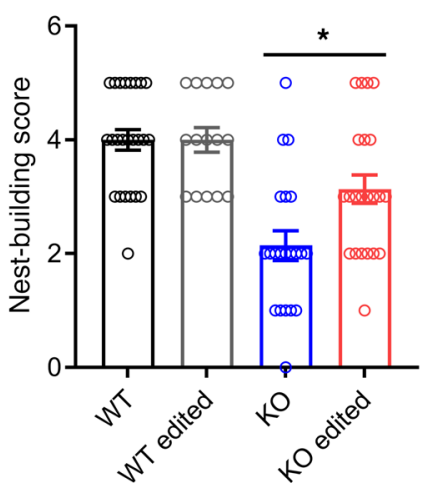

B

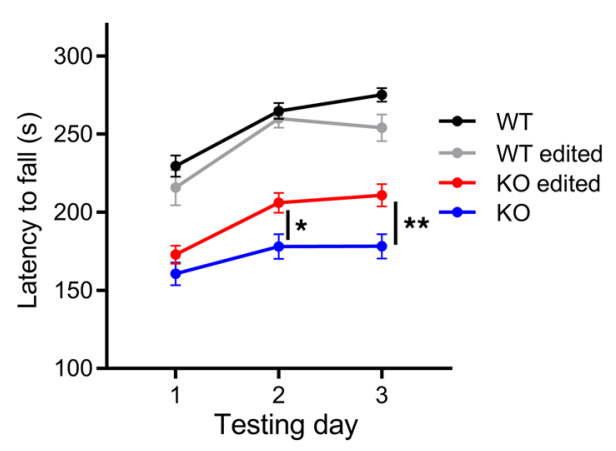

E

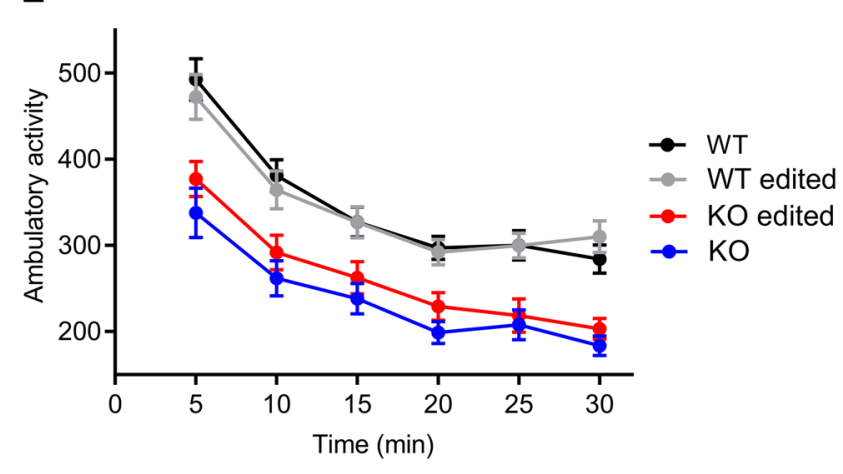

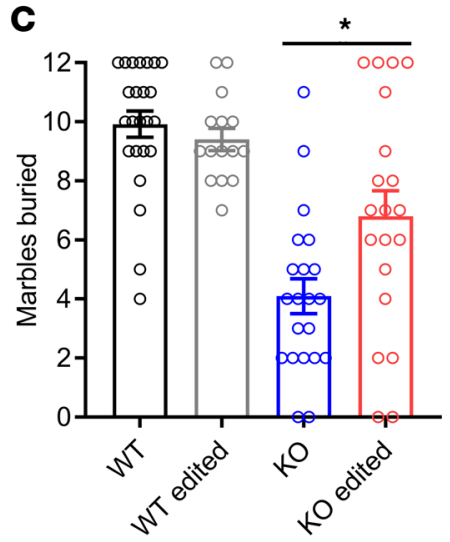

Figure 3. Phenotypic improvement in an AS mouse model after gene editing. Ube $3 a^{m-/ p+}$ and Ube $3 a^{m+/ p+}$ littermates received a neonatal injection of ATS-GE or control vector. (A) Mouse weight increased over the observation period but showed no group effect $(\mathrm{F}[1.311,64.25]=385.3, P>0.2, n=15$ mice/ group). (B) At 8 weeks of age, we tested motor function with a rotarod apparatus over 3 consecutive days. On days 2 and 3 , gene-edited Ube3am-/p+ mice showed a significant motor improvement compared with Ube3am-/p+ mice that received control vector (2-way ANOVA with Tukey's pairwise comparison, $n=15-24$ mice/group). (C) Gene-edited Ube3 $a^{m-/ p+}$ mice demonstrated a significant improvement in burying activity compared with $U b e 3 a^{m-/ p+}$ mice that received control vector (1-way ANOVA with Tukey's pairwise comparison, $n=15-24$ mice/group). (D) Cene-edited Ube3am-pp+ mice demonstrated a significant improvement in nest-building skills and activity compared with Ube3am-/p+ mice that received control vector (1-way ANOVA with Tukey's pairwise comparison, $n=15-24$ mice/group). (E) We assessed the same mouse cohort in an open-field arena to determine ambulatory activity. The performance of the gene-edited Ube $3 a^{m+/ p-}$ mice showed a trend of improvement at all timepoints compared with Ube3a ${ }^{m+/ p-}$ mice that had received the control vector ( $n=15-24$ mice/group, 2-way ANOVA: treatment group effect $(F[3,77]=13.48, P<0.001)$; Tukey's pairwise comparison, $P>0.4)$. Means are shown with standard error, ${ }^{*} P<0.05,{ }^{* *} P<0.01$.

(AMP-Seq, 2.1\%, Supplemental Figure 4C) as previously observed (Supplemental Figure 2A). Off-target analysis by ITR-Seq did not show an increased rate of off-target effects (Supplemental Table 4) compared with the previous short-term study (Supplemental Table 3). The only 2 identified off-target sites were located in an intron or an unannotated genomic region. ATS-Ube3a transcript levels were significantly reduced in Ube3a-KO mouse brains after gene editing (Figure 2G). The transcript levels were found to have normalized about $4 \mathrm{~kb}$ from the gene-editing site toward the imprinting center.

The behavioral phenotype of maternal Ube3a-KO mice has been well characterized and can be improved by genetically restoring maternal Ube3a expression. Treatment with ASOs transiently suppresses the extension of Ube3a-ATS across the Ube3a locus, leading to paternal Ube3a expression in neurons throughout the brain and the subsequent improvement of the behavioral phenotype. This approach restores Ube3a expression in a much larger number of neurons throughout the mouse brain, so we were wondering whether gene editing of Ube3a-ATS in a limited number of neurons could improve the maternal Ube3a-KO phenotype. We i.c.v.-injected neonatal mice with ATS-GE vector and monitored them until 4 months of age. Ube3a-ATS gene editing was tolerated well with no treatment-related mortalities. As expected, weight gain was significantly higher in AS mice and showed a trend to reduction after ATS-GE treatment during the observation period (Figure 3A). At 2 months of age, the mice were subjected to a sequence of behavioral tests that have been widely used with this mouse model (17). Maternal Ube3a-KO mice showed the expected significant deficits in motor function in comparison to their WT littermates when tested with a rotarod (Figure 3B). Gene-edited maternal Ube3a-KO mice showed a significant improvement of motor function on testing days 2 and 3 (Figure 3B). Similarly, marble burying and nest-building behaviors were impaired in maternal Ube3a-KO mice, but were significantly improved after Ube3a-ATS gene editing (Figure 3, C and D). Ambulatory activity in the openfield test showed a modest yet consistent trend toward improvement of hypoactivity in maternal Ube3a-KO mice after Ube3a-ATS gene editing (Figure 3E). In summary, restoring Ube3a expression by suppressing Ube3a-ATS in a subset of neurons significantly improved multiple behavioral aspects of the maternal Ube3a-KO phenotype. It remains to be investigated whether Ube3a-ATS gene 
editing improves other maternal Ube3a-KO mouse phenotypes, such as electroencephalogram alterations.

This study demonstrated two important findings: (a) efficient gene editing can be achieved in the mouse brain by neonatal intraventricular AAV delivery and (b) expression of Ube3a in a subset of neurons (a maximum of $20 \%$ based on sequencing data) provided a therapeutic benefit in an AS mouse model. Nuclease activity of Cas9 was necessary to achieve Ube3a protein expression since expression of inactive Cas9 (dCas9) did not cause Ube3a protein expression.

The CNS has been recognized as a promising target for therapeutic genome editing, particularly since disruption of a pathological allele may represent a curative treatment of genetic disorders (18-20). Recent studies for therapeutic CNS gene editing have achieved promising results via focal delivery of CRISPR/Cas9 complex, e.g., into the striatum of a Huntington's disease mouse model (21), into the spinal cord of an ALS mouse model (22), or into the hippocampus of a mouse model of familial Alzheimer's disease (23). However, it remains to be shown whether CRISPR/Cas9 can successfully edit a sufficient number of neurons to achieve a therapeutic benefit in human patients if editing throughout different brain regions needs to be achieved. Our study demonstrated that this is possible in the mouse brain and can result in the significant improvement of a disease phenotype. For AS, as for many other genetic CNS disorders, we know little about the required efficiency of disease gene reexpression to cause a therapeutic benefit. Our study suggests that reexpression of Ube3a is not required in all neurons. Previous studies that used genetic reinstatement of Ube3a or ASO-mediated Ube3a expression showed a larger effect size of behavior improvement due to the much larger number of neurons expressing Ube3a $(8,11,12)$. Our studies are encouraging for further translational and clinical AS research since inefficient expression of UBE3A may already be enough to translate into a therapeutic effect in patients. A recent study with CRISPR/Cas9-mediated replacement of a $245 \mathrm{~kb}$ section within the Ube3a-ATS with an AAV vector insertion also reported limited neurobehavioral improvement in AS model mice (24).

Our study does not directly address a potential mechanism for how indel formation results in a shortened Ube3a-ATS that selectively enables lasting paternal Ube3a expression. We can only speculate that the secondary or tertiary genomic structure of the Ube $3 a$ locus may play a role in the antagonistic gene expression regulation of Ube3a and Ube3a-ATS. Alternatively, or additionally, we speculate that the extremely long paternal Ube3a-ATS transcript may be prone to early termination in the presence of the paternal sense Ube $3 a$ transcript. Gene editing may cause an initial transcriptional pausing of the Ube3a-ATS transcript, which allows formation of the Ube3a sense transcript, with this situation persisting even after double-strand repair has been completed. Another possible mechanism of action includes the ability of S. aureus Cas9 to cleave RNA transcripts (25), which likely would require constitutive expression of Cas9 to maintain Ube3a expression; we did not, however, observe sustained Cas9 expression in all Ube3aexpressing AS mouse brains (data not shown). Lastly, integration of AAV vector sequence could lead to premature termination of Ube3a-ATS, as observed in a recent study (24). Given that total detected integrations remained at $2 \%$ to $3 \%$ over 4 months, this mechanism could contribute to, but is unlikely to be solely responsible for, all detected Ube3a expression.

Although the genomic organization and regulation of Ube3aATS and the imprinting control center are highly conserved between mouse and human (11), the DNA sequence is very different for these two species. A redesign of the mouse sgRNA targeting a similar genomic location in humans would be a necessary prerequisite for translational studies. One would expect that interference of UBE3A-ATS by gene editing could similarly restore UBE3A expression in the neurons of patients with AS. However, additional safeguards to limit Cas9 activity beyond the initial gene-editing event may be necessary to prevent unintended off-target activity. We are cautiously optimistic that this approach may represent a potential long-lasting therapeutic option for patients with AS.

\section{Methods}

For additional information, see Supplemental Methods.

Data availability. All high-throughput sequencing data sets are available at https://www.ncbi.nlm.nih.gov/bioproject/PRJNA686778.

Study approval. All animal protocols and procedures were approved by the University of Pennsylvania IACUC (Animal Welfare Assurance Number A3079-01). The animals' housing facility was accredited by AAALAC.

\section{Author contributions}

RSS and JMW conceived and designed the experiments. RS, PP, $\mathrm{XD}, \mathrm{MM}$, and $\mathrm{CB}$ performed the experiments. RS, $\mathrm{PP}, \mathrm{XD}, \mathrm{MM}$, $\mathrm{CB}$, and JMW analyzed the data. RS and JMW wrote the manuscript. All authors read and approved the manuscript.

\section{Acknowledgments}

We thank Koita Sacko and Nathan Denton for their assistance. We thank the Vector Core, Program in Comparative Medicine, Histology Core, and Nucleic Acid Technologies Core within the University of Pennsylvania Gene Therapy Program. This study was funded by the Gene Therapy Program and the Foundation for Angelman Syndrome Therapeutics.

Address correspondence to: James M. Wilson, Gene Therapy Program, University of Pennsylvania Perelman School of Medicine, Philadelphia, Pennsylvania, USA. Phone: 215.573.9020; Email: wilsonjm@upenn.edu.
1. Buiting K, et al. Angelman syndrome - insights into a rare neurogenetic disorder. Nat Rev Neurol. 2016;12(10):584-593.

2. Dagli A, et al. Molecular and clinical aspects of Angelman syndrome. Mol Syndromol. 2012;2(3-5):100-112.
3. Albrecht $\mathrm{U}$, et al. Imprinted expression of the murine Angelman syndrome gene, Ube3a, in hippocampal and Purkinje neurons. Nat Genet. 1997;17(1):75-78.

4. Kishino T, et al. UBE3A/E6-AP mutations cause Angelman syndrome. Nat Genet. 1997;15(1):70-73.
5. Matsuura T, et al. De novo truncating mutations in E6-AP ubiquitin-protein ligase gene (UBE3A) in Angelman syndrome. Nat Genet. 1997;15(1):74-77.

6. Keute M, et al. Angelman syndrome genotypes manifest varying degrees of clinical severity and developmental impairment [published online 
August 13, 2020]. Mol Psychiatry. https://doi. org/10.1038/s41380.020.0858-6.

7. Chamberlain SJ, Brannan CI. The Prader-Willi syndrome imprinting center activates the paternally expressed murine Ube3a antisense transcript but represses paternal Ube3a. Genomics. 2001;73(3):316-322.

8. Meng L, et al. Truncation of Ube3a-ATS unsilences paternal Ube3a and ameliorates behavioral defects in the Angelman syndrome mouse model. PLoS Genet. 2013;9(12):e1004039.

9. Rougeulle C, et al. An imprinted antisense RNA overlaps UBE3A and a second maternally expressed transcript. Nat Genet. 1998;19(1):15-16.

10. Huang HS, et al. Topoisomerase inhibitors unsilence the dormant allele of Ube3a in neurons. Nature. 2012;481(7380):185-189.

11. Meng L, et al. Towards a therapy for Angelman syndrome by targeting a long non-coding RNA. Nature. 2015;518(7539):409-412.

12. Silva-Santos $S$, et al. Ube3a reinstatement identifies distinct developmental windows in a murine Angelman syndrome model. J Clin Invest.
2015;125(5):2069-2076.

13. Kügler S, et al. Human synapsin 1 gene promoter confers highly neuron-specific long-term transgene expression from an adenoviral vector in the adult rat brain depending on the transduced area Gene Ther. 2003;10(4):337-347.

14. Thiel G, et al. Characterization of tissue-specific transcription by the human synapsin I gene promoter. Proc Natl Acad Sci U S A. 1991;88(8):3431-3435.

15. Deverman BE, et al. Cre-dependent selection yields AAV variants for widespread gene transfer to the adult brain. Nat Biotechnol. 2016;34(2):204-209.

16. Dindot SV, et al. The Angelman syndrome ubiquitin ligase localizes to the synapse and nucleus, and maternal deficiency results in abnormal dendritic spine morphology. Hum Mol Genet. 2008;17(1):111-118.

17. Sonzogni M, et al. A behavioral test battery for mouse models of Angelman syndrome: a powerful tool for testing drugs and novel Ube $3 a$ mutants. Mol Autism. 2018;9:47.

18. van Haasteren J, et al. The delivery challenge: fulfilling the promise of therapeutic genome editing. Nat Biotechnol. 2020;38(7):845-855.

19. Cox DB, et al. Therapeutic genome editing: prospects and challenges. Nat Med. 2015;21(2):121-131.

20. Nishiyama J. Genome editing in the mammalian brain using the CRISPR-Cas system. Neurosci Res. 2019;141:4-12.

21. Ekman FK, et al. CRISPR-Cas9-mediated genome editing increases lifespan and improves motor deficits in a Huntington's disease mouse model. Mol Ther Nucleic Acids. 2019;17:829-839.

22. Gaj $\mathrm{T}$, et al. In vivo genome editing improves motor function and extends survival in a mouse model of ALS. Sci Adv. 2017;3(12):eaar3952.

23. Gyorgy B, et al. CRISPR/Cas9 mediated disruption of the Swedish APP allele as a therapeutic approach for early-onset Alzheimer's disease. Mol Ther Nucleic Acids. 2018;11:429-440.

24. Wolter JM, et al. Cas9 gene therapy for Angelman syndrome traps Ube3a-ATS long non-coding RNA. Nature. 2020;587(7833):281-284.

25. Strutt SC, et al. RNA-dependent RNA targeting by CRISPR-Cas9. Elife. 2018;7:e32724. 